\title{
Fractal to Euclidean crossover and scaling for random walkers on percolation clusters
}

\author{
P. Argyrakis ${ }^{\text {a) }}$ \\ Department of Physics, University of Crete, Iraklion, Crete, Greece \\ R. Kopelman b) \\ Department of Chemistry, The University of Michigan, Ann Arbor, Michigan 48109
}

(Received 14 February 1984; accepted 4 April 1984)

\begin{abstract}
Simulations of random walkers on two-dimensional (square lattice) percolation clusters were performed for a range of occupation probabilities from critical to unity. The number of distinct sites visited, over $2 \times 10^{5}$ steps, shows the conjectured scaling, crossover and superuniversality $\left(d_{s}=4 / 3\right.$, within $\left.1 \%\right)$ behavior over a wide range of site occupation probabilities. Possible deviations from superuniversality and/or scaling are discussed.
\end{abstract}

\section{INTRODUCTION}

The idea that the random walk efficiency on a percolating cluster increases drastically with increasing occupied site concentration is nearly a decade old. ${ }^{1}$ The number of distinct sites visited and the trapping probability of the walker were first simulated by Argyrakis, Hoshen, and Kopelman..$^{2-4}$ The mean square displacement of an "ant in a labyrinth" was first discussed by de Gennes ${ }^{5}$ and related to the critical correlation exponent $(v)$. While early diffusion simulations seemed to support this, ${ }^{6}$ later work ${ }^{7}$ showed the superiority of the scaling approach. ${ }^{8}$ However, a deeper understanding of the subject occurred only a year ago, with the realization that random walk on critical percolating clusters is controlled by a fractal spectral ("fracton") dimension..$^{9,10}$ It was realized soon thereafter that crossovers must exist between the fractal and the Euclidean behavior and that this crossover must be related to the correlation length. ${ }^{11-14}$ Such crossovers are indicated in the Monte Carlo simulations of the mean square displacements by Havlin et al. ${ }^{13}$ and even in the much older simulations. ${ }^{15}$ We show here the results of high quality simulations on the number of distinct sites visited for a single walker (elsewhere we show similar simulations for the density of reacting random walkers, i.e., reactions of multiple random walkers, in two and three dimensions). ${ }^{28}$ These results are not only of theoretical interest, but are related to various experiments on diffusion, trapping, and annihilation (fusion) reactions, especially for excitons on isotopic mixed crystals. ${ }^{15-22}$ Crossovers from fractal to Euclidean behavior are already implicit in our old simulations of single walkers on random lattices ${ }^{1,4,23}$ and of multiple (reacting) walkers. ${ }^{24-28}$ Here we present a detailed study, over $2 \times 10^{5}$ steps, of such fractal-to-Euclidean crossovers for single random walkers on percolating clusters of square lattices.

The average number of distinct sites visited $S_{N}$, after $N$ steps on a percolation cluster, at criticality, is given by

$$
S_{N} \propto N^{d, 2}, \quad N \rightarrow \infty,
$$

where $d_{s}$ is the spectral dimension, ${ }^{9,10}$ which is believed to be $4 / 3$, i.e., the exponent in Eq. (2) is $2 / 3$, for values of $p=p_{c}$, where $p$ is the probability and $p_{c}$ is its value at the critical percolation point. In the spirit of Gefen et al. ${ }^{11}$ and Havlin et al..${ }^{13}$ we write

$$
S_{N}(t \rightarrow \infty, p)=t^{d_{j} / 2} f\left[\left(p / p_{c}-1\right) t^{-(2 v-\beta+\mu)}\right] .
$$

This scaling relation includes the well-known static percolation exponents ${ }^{8}$ for the correlation length $(v)$, the percolation probability $(\beta)$ and the conductivity $(\mu)$. We check here the range of validity of $\mathrm{Eq}$. (2). We note that the crossover time * $^{*}$ (from fractal-to-Euclidean) is given ${ }^{11,13}$ by

$$
t^{*} \propto\left|p-p_{c}\right|^{-(2 v-\beta+\mu)} .
$$

The preexponent can be found, in principle, from our simulation results. On the other hand, we try a crude approach

$$
S_{N} \propto t^{d_{s}^{\prime} / 2},
$$

where $d_{s}^{\prime}$ is an effective spectral dimension ${ }^{29}$ (with values between 0 and 2). While the scaling approach works up to $p=0.7$, the crude approach works throughout the crossover regime and is directly applicable to experiments. ${ }^{15-22}$

\section{METHOD OF CALCULATIONS}

We use Monte Carlo simulation techniques, which are in the spirit of the model recently reported, ${ }^{30}$ but have considerably improved the algorithm, increasing both the speed of operations and the size of the lattice used. A binary lattice is made of two components that are termed as either allowed, open sites labeled 1 , or nonallowed, closed sites labeled 0 . All 1 's and 0 's are mixed at random, for any given ratio. All random walks take place only on connected open sites, with zero probability for a step to a closed site. In previous practice, all lattices were generated and stored in computer memory before the start of each run and remained active for the whole duration of the execution, something rather expensive because lattice portions that were never used still occupied a considerable memory space. The present algorithm, called the cluster growth technique, generates only the lattice portions used for the random walk, thus effectively increasing the size of the lattice. The lattice starts with one site only, and

\footnotetext{
2) Supported by NATO Grant No. SA 5205 RG 295/83.

${ }^{\text {b) }}$ Supported by NSF Grant No. DMR 8303919.
} 
it is built continuously as the random walk proceeds by generating more sites adjacent to the random walker. Of course, once a site is generated and its identity chosen it remains as such in the memory for the whole run. Obviously, only one random walk can be executed on each lattice using this method, which is more time consuming but has considerable advantages from a statistical point of view.

Since only five alternative pieces of information are needed at each time (the identity of the site, 0 or 1 , and whether a site has been visited before or not, or whether it has not been defined), we can utilize more efficiently the length of each computer word by breaking each 32 bit word in four 8 bit sections, thus effectively increasing the size of memory (direct access) by a factor of 4 . This is done in FORTRAN by use of a subroutine with LOGICAL*1 and INTEGER $* 4$ variables declaration, occupying the same memory space and identified continuously via an EQUIVALENCE statement. We thus avoid the difficult task of byte manipulation in machine language. The outcome is that the lattice size $L$ is now larger, typically in the vicinity of $L=4 \times 10^{6}$ sites. Our aim has been to monitor a random walk process that never needs to cross the boundary sites, so that in practice our results pertain to an infinite lattice. We let $N=200000$ be our maximum value so that $N / L=0.05$, which is a very safe ratio. In the past, where high $N / L$ ratios were used (of the order of 1 , or even higher), it was found necessary to use alternate indirect methods to extrapolate to the case of an infinite lattice. The problem that arises in the case of high $N / L$ ratio is that of artificial revisitation, where the walker is confined to a small region, due to the small $L$ producing a smaller $S_{N}$ value than the normal.

All different topologies in one-, two-, and three-dimensional lattices are reduced to a one-dimensional array in the virtual computer memory, thus saving on transfer times and increasing the speed of operations. All calculations are performed in a DEC computer model VAX 11/750 with 550 Mbytes of total memory. We use alternate random number generation routines by IBM, DEC, and by the University of Michigan MTS Computer Center to ensure absolute randomness of events.

\section{RESULTS AND DISCUSSION}

Figure 1 shows the random walk crossover for the square lattice site percolation case. Close to the critical point $(p=0.593) S_{N}$ has a simple power dependence on the time (number of steps $N$ ) and, using Eq. (1), $\log S_{N}$ is linear with $\log N$. The very small corrections to the asymptotic behavior of Eq. (1) are analyzed elsewhere. ${ }^{30}$ An effectively simple power dependence is again seen for the $p=1.0$ case (in spite of the well-known logarithmic correction for this $2 D$ lattice). However, for all intermediate values of $p$ the lines are curved (upward!), showing the crossover from fractal to Euclidean behavior.

Figure 2 is a scaling plot, based on Eq. (2) with $d_{s}=4 / 3$, according to the superuniversality conjecture. ${ }^{9-11}$ We also use $v=4 / 3, \beta=5 / 36$ according to the den Nijs conjecture (proved now?) and $\mu=(1 / 2)[v(3 d-4)-\beta]$, again according to the $d_{S}=4 / 3$ conjecture, giving, for $d=2, \mu=91 / 72$. Hence, $2 v-\beta+\mu=91 / 24 \approx 3.79$. Using instead of the

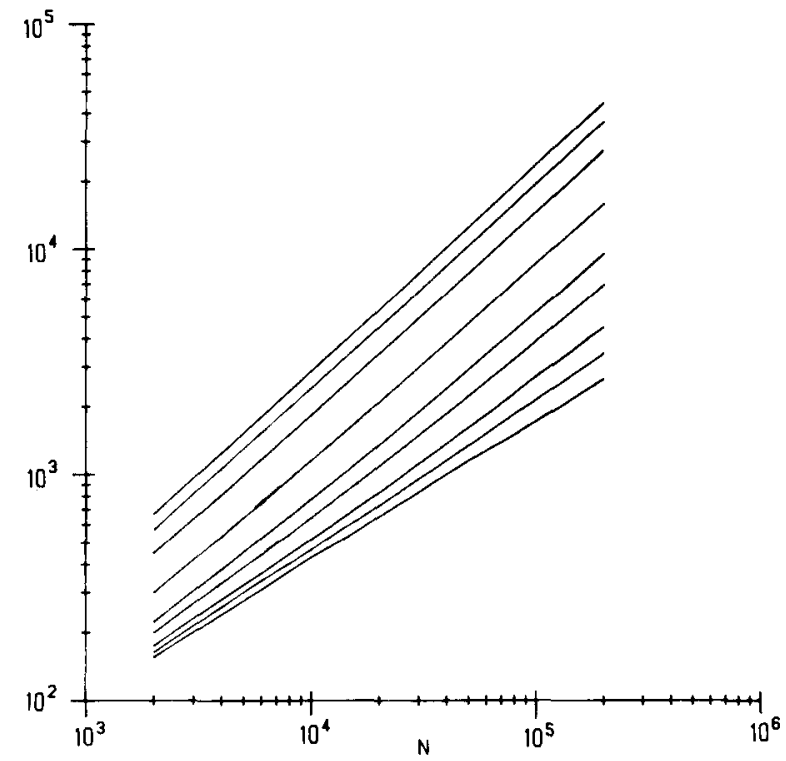

FIG. 1. $S_{N}$ vs $N$ for binary square lattices. Walk on percolating cluster only (see Refs. 29 and 30). Top to bottom: $p=1.0,0.9,0.8,0.7,0.65,0.63,0.61$, 0.60 , and 0.59 .

above value (3.79) values of 3.6 and 4.0 yielded slightly inferior scaling plots (as judged by the eye). We note that the Fig. 2 plot is quite satisfactory if we remember that our simulation included 200000 steps (compared to 1600 by Havlin et al. ${ }^{13}$ for the scaling of the diffusion, i.e., mean square displacement) and that for our case of $S_{N}$ all relevant exponents are exact, with no room for adjustable parameters.

Figure 3 shows that the scaling behavior does fail further out from the critical point, as expected. While for $p=0.7$ scaling still seems to work, this is not the case for $p=0.8$ or higher. This result is again consistent with that of Havlin et al. ${ }^{13}$ even though the latter authors do not show results for $p>0.7$ and also, as stated above, their time do-

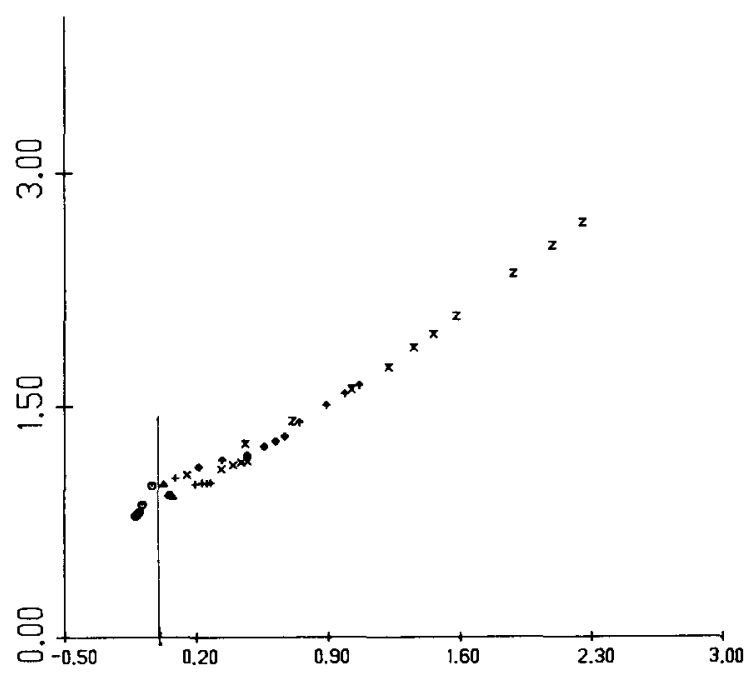

FIG. 2. Scaling plot: $S_{N} / N^{2 / 3} v s\left(p / p_{c}-1\right) N^{0.263}$, for walk on percolating cluster. The different symbols correspond to different $p$ values: $0.59,0.595$, $0.60,0.605,0.61,0.62,0.63$, and 0.65 . Note that the $p=0.59$ values are below the threshold and, thus, appear to the left of the line $X=0$. The $N$ values picked are: $2000,42000,82000,122000$, and 162000 . 


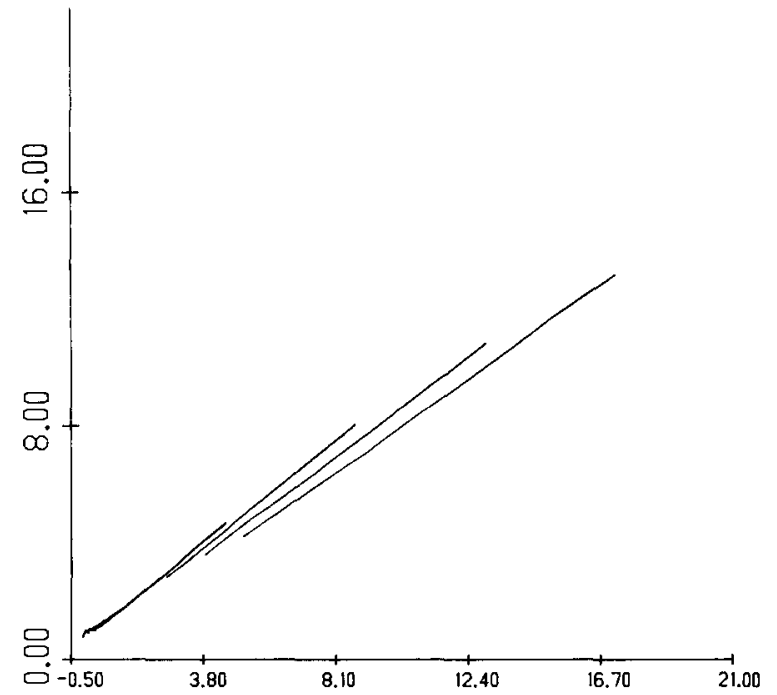

FIG. 3. Extended scaling plot: $S_{N} / N^{2 / 3}$ vs $\left(p / p_{c}-1\right) N^{0.263}$. Same as Fig. 2 (contracted) but with the addition of curves for $p=0.7,0.8,0.9$ and 1.0 (left to right). Note that the $p=0.7$ curve merges with the lower $p$ scaling curves of Fig. 1. However, the $p=0.8,0.9$, and 1.0 curves seem to deviate from it.

mains are three orders of magnitude shorter. Thus, it appears that the correlation length $(\xi)$ is a useful concept in the range $0.593 \leqslant p \leqslant 0.7$, as is the corresponding exponent $(v)$. Qualitatively, these results are consistent with the behavior (curvature) of $S_{N}$ vs $N$ in Fig. 1.

Figures 4 and 5 show the behavior of the effective fractal exponent. While above the critical point $\left(p_{c}\right)$ the crossover is in the direction of the Euclidean (classical) behavior (and slope), this is not so below $p_{c}$, where the "crossover" is towards the random walk on a finite cluster behavior, resulting in an effective "saturation" of the sites (all are visited).

Figure 6 is another representation of the same qualitative ideas shown in Figs. 4 and 5, i.e., an effective spectral dimension. It is obvious, that for longer times $\left(N \approx 2 \times 10^{5}\right)$, the rise of $d^{\prime}{ }_{s}$ is sharper than at short times. This means that for $p \simeq p_{c}$ the random walk becomes more "efficient." This plot itself reminds us of similar plots relating to the trapping

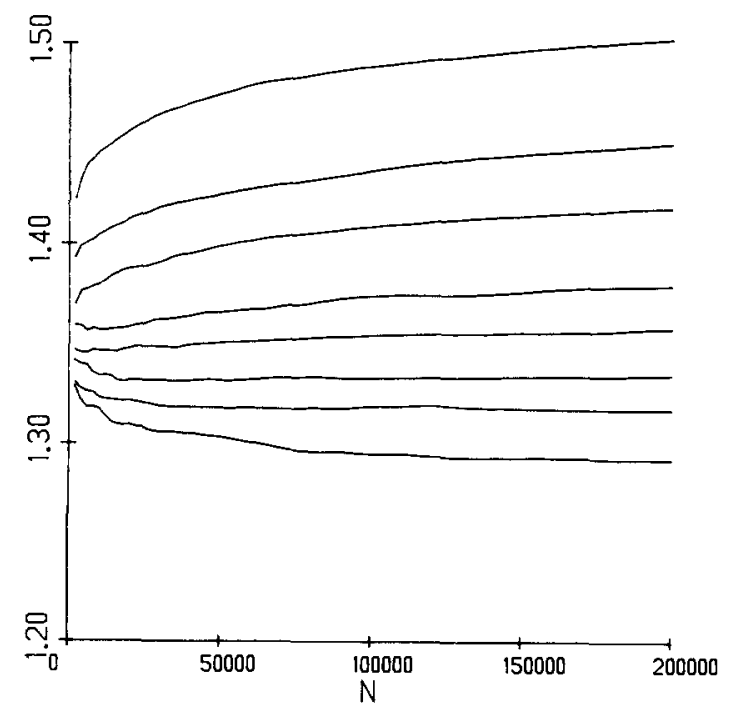

FIG. 4. $2 \log S_{N} / \log N$ vs $N$ for largest cluster walk. Top to bottom: $p=0.65,0.63,0.62,0.61,0.605,0.60,0.595$, and 0.59 .

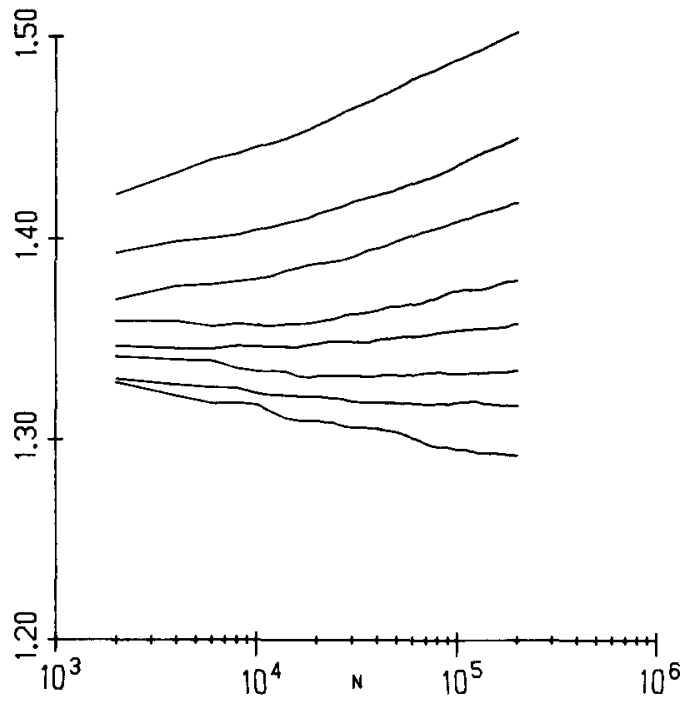

FIG. $5.2 \log S_{N} / \log N$ vs $N$, same as Fig. 4, but semilogarithmic representation.

of random walkers (excitons) in both experiments and simulations. ${ }^{1-4,16-23}$ Obviously, for shorter times, the effective $d^{\prime}{ }_{s}$ rises less sharply with $p$. This just means that for times shorter than the correlation time ["crossover time"-Eq. (4)] the random walk is essentially "fractalized," i.e., the correlation length is longer than the path of the random walker. We also notice that for the naphthalene crystal the singlet exciton is believed to hop about $2 \times 10^{5}$ times within its lifetime. ${ }^{2,16}$ Thus, the simulations given here should be applicable to past and future trapping and annihilation experiments.

Finally, we would like to point out that the present study does not provide a stringent test for the value of $\mu$. A

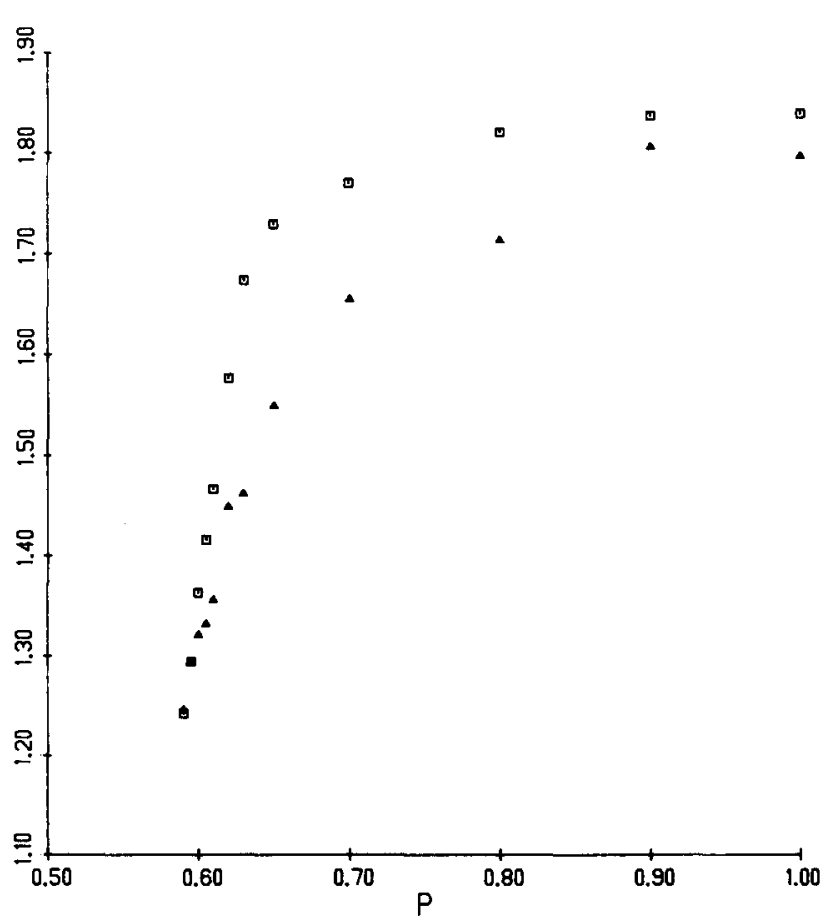

FIG. 6. $d^{\prime}{ }_{s}$ vs $p$ for walk on percolating cluster. Squares-from long time limit $(N=200000 / N=100000)$. Triangles-from short time limit $(N=4000 / N=2000)$. 
0.2 uncertainty in the reciprocal crossover exponent (see above) would give a $15 \%$ uncertainty in $\mu$. Recent direct simulations ${ }^{31}$ on $\mu$ have claimed a value of about $3 \%$ above 91/72. We cannot dispute that. However, if we believe the Alexander and Orbach scaling relation ${ }^{9-11}$

$$
\mu-\beta+2 v=2 d_{s}(d v-\beta),
$$

then this would result in $d_{s}$ being $1 \%$ below $4 / 3$. This would just be consistent with our present best statistical evaluation of the long time random walk, just above criticality ${ }^{32}$ $d_{s} / 2=0.66 \pm 0.01$, i.e., nominally $1 \%$ below $2 / 3$. Thus, there seems to be no problem with the above scaling relationship, so far, and the suspected correction to superuniversality, in two dimensions, is about $1 \%$ downwards, for $d_{s}$.

In summary, our long time random walk simulations confirm the scaling, crossover, and superuniversality hypothesis of random walkers on percolation clusters to within a few percent. Furthermore, they show a fairly wide validity range for the scaling behavior and a consistency between the crossover exponent and the superuniversality conjecture $\left(d_{s}=4 / 3\right)$. Similar studies for three-dimensional lattices are in progress.

\section{ACKNOWLEDGMENTS}

We would like to thank S. Alexander, S. Havlin, J. Hoshen, J. Klafter, R. Orbach, and I. Webman for helpful discussions.

'R. Kopelman, in Proceedings of the Seventh Molecular Crystal Symposium (Nikko, Japan, 1975), p.37.

${ }^{2}$ R. Kopelman, in Topics in Applied Physics, edited by F. K. Fong (Springer,
Berlin, 1976), Vol. 15, p. 297.

${ }^{3}$ J. Hoshen and R. Kopelman, J. Chem. Phys. 65, 2817 (1976)

${ }^{4}$ P. Argyrakis, Ph.D. thesis, University of Michigan, 1978.

${ }^{5}$ P. G. de Gennes, Recherche 7, 919 (1976).

${ }^{6}$ C. D. Mitescu and J. Rousseng, C. R. Acad. Sci. Paris A 283, 999 (1976).

${ }^{7}$ T. Vicsek, Z. Phys. B 45, 153 (1981).

${ }^{8}$ D. Stauffer, Phys. Rep. 54, 1 (1979).

${ }^{9}$ S. Alexander and R. Orbach, J. Phys. Lett. 43, L625(1982).

${ }^{10}$ R. Rammal and G. Toulouse, J. Phys. Lett. 44, L13 (1983).

"Y. Gefen, A. Aharony, and S. Alexander, Phys. Rev. Lett. 50, 77 (1983).

${ }^{2}$ P. G. de Gennes, C. R. Acad. Sci. Paris 296, 881 (1983).

${ }^{13}$ S. Havlin, D. Ben Avraham, and H. Sompolinsky, Phys. Rev. A 27, 1730 (1983).

${ }^{14}$ I. Webman, Phys. Rev. Lett. 52, 220(1984).

${ }^{15} \mathrm{C}$. D. Mitescu, H. Ottavi, and J. Roussenq, in Electrical Transport and Optical Properties of Inhomogeneous Media-1977, edited by J. C. Garland and D. B. Tanner (American Institute of Physics, New York, 1978).

${ }^{16}$ P. Argyrakis and R. Kopelman, J. Chem. Phys. 66, 3301 (1977).

${ }^{17}$ P. Argyrakis and R. Kopelman, Chem. Phys. Lett. 61, 187 (1979).

${ }^{18}$ P. W. Klymko and R. Kopelman, J. Lumin. 24, 25, 457 (1981).

${ }^{19}$ P. Argyrakis and R. Kopelman, Chem. Phys. 57, 29 (1981).

${ }^{20}$ P. W. Klymko and R. Kopelman, J. Phys. Chem. 86, 3686 (1982).

${ }^{21}$ P. Argyrakis and R. Kopelman, Chem. Phys. 78, 251 (1983).

${ }^{22}$ P. W. Klymko and R. Kopelman, J. Phys. Chem. 87, 4565 (1983).

${ }^{23}$ P. Argyrakis and R. Kopelman, J. Theor. Bio. 73, 205 (1978).

${ }^{24} \mathrm{P}$. Argyrakis, J. Hoshen, and R. Kopelman, in Fast Reactions in Energetic Systems, edited by C. Capellos, R. F. Walker, and D. Reidel (Dordrecht, Holland, 1980), p.685.

${ }^{25}$ R. Kopelman, J. Hoshen, J. S. Newhouse, and P. Argyrakis, J. Stat. Phys. 30, 355 (1983).

${ }^{26}$ R. Kopelman, P. W. Klymko, J. S. Newhouse, and L. Anacker, Phys. Rev. B 29, 3747 (1984).

${ }^{27}$ L. Anacker, R. Kopelman and J. S. Newhouse, J. Stat. Phys. (1984).

${ }^{28} \mathrm{~J}$. S. Newhouse, P. Argyrakis, and R. Kopelman, Chem. Phys. Lett. (in press).

${ }^{29} \mathrm{P}$. Argytkias and R. Kopelman, Phys. Rev. B 29, 511 (1984).

${ }^{30}$ A. I. Keramiotis, P. Argyrakis, and R. Kopelman, Phys. Rev. B (in press).

${ }^{31} R$. Orbach (private communication). We also thank the referee for bringing similar, unpublished, results to our attention.

${ }^{32}$ L. Anacker, P. Argyrakis, and R. Kopelman, J. Stat. Phys. (in press). 